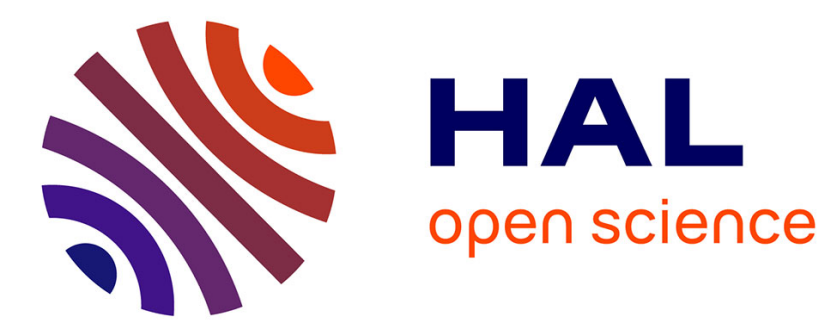

\title{
Benchmark solutions and assessment of variable kinematics models for global and local buckling of sandwich struts
}

\author{
M. d'Ottavio, O. Polit, W. Ji, A.M. Waas
}

\section{- To cite this version:}

M. d'Ottavio, O. Polit, W. Ji, A.M. Waas. Benchmark solutions and assessment of variable kinematics models for global and local buckling of sandwich struts. Composite Structures, 2016, to appear, pp.125-134. 10.1016/j.compstruct.2016.01.019 . hal-01366896

\section{HAL Id: hal-01366896 https://hal.science/hal-01366896}

Submitted on 11 Jan 2019

HAL is a multi-disciplinary open access archive for the deposit and dissemination of scientific research documents, whether they are published or not. The documents may come from teaching and research institutions in France or abroad, or from public or private research centers.
L'archive ouverte pluridisciplinaire HAL, est destinée au dépôt et à la diffusion de documents scientifiques de niveau recherche, publiés ou non, émanant des établissements d'enseignement et de recherche français ou étrangers, des laboratoires publics ou privés. 


\title{
Benchmark solutions and assessment of variable kinematics models for global and local buckling of sandwich struts
}

\author{
Michele D’Ottavio, Olivier Polit, Wooseok Ji, Anthony M. Waas
}

\section{Introduction}

Sandwich panels basically consist of two thin and stiff load carrying face sheets (the skins) that are separated by a thick and relatively weak core made of low density material. The particularly high specific bending stiffness renders sandwich panels an appealing load bearing solution in designs with relevant weight constraints. Failure mechanisms of sandwich panels can be very different from those of other composite structures due to the characteristic mismatch of mechanical and geometrical properties between skins and core [1,2]. In particular, instability phenomena occurring under compressive stress states have been given great attention through numerous experimental works [3-6] as well as theoretical and computational developments [7-15].

Compression instabilities in sandwich panels can be classified with respect to the wavelength of the buckling pattern [16]: face dimpling denotes the intracellular face buckling within honeycomb cells; wrinkling is characterized by a wavelength that is comparable to the face thickness; overall or global buckling identifies instabilities with wavelengths of the same order of magnitude of the panel in-plane dimension. These global and local instabilities may interact and form a complex failure scenario [17-20]. The differences between the length scales of the response demand appropriate modeling techniques [21,22].
The classical manner to compute buckling instabilities refers to a linearized bifurcation analysis that permits to determine critical loads by a simple eigenvalue analysis, and the present work follows this approach also. The authors are aware that the loads predicted by this simplified analysis should be reduced by opportune knockdown factors in order to account for the imperfection sensitivity of the instability process [23]. The alternative to the linearized bifurcation analysis is the solution of the full nonlinear equilibrium and stability problem, including initial perturbations of geometrical and/or mechanical nature [9,24,25].

When dealing with the linearized bifurcation analysis great attention should be given to the definition of the incremental strain and stress measures that describe the adjacent equilibrium state. Bažant [26] demonstrated the equivalence of formulations based on different incremental strain and work-conjugate incremental stress measures provided the corresponding constitutive equations is used, see also [27]. The selection of a consistent set of relations defining the incremental strains and stresses and the constitutive model is of utmost importance when dealing with soft, shear-flexible materials like those usually employed in sandwich structures [28,29]. A quantitative assessment of different incremental elasticity formulations has been provided by comparing the buckling loads obtained from exact elasticity solutions [30] or accurate FE analyses [31] for various sandwich (wide) beams. They showed that these inconsistencies may lead to errors of up to $20 \%$ and that the erroneous results are non-conservative. 
Despite elasticity solutions of the linear bifurcation problem of sandwich panels are available for a restricted class of problems [30,32-34], it is often necessary to resort to approximate structural models in order to cope with more complex configurations [35]. Many plate and shell models have indeed been proposed for sandwich panels, as already summarized by the reviews of Noor and Burton [36,37]. Carrera [38,39] paved the way for a very efficient manner to assess the accuracy of different models. Thanks to a compact index notation, variable kinematic models are formulated and the corresponding governing equations are implemented in a unique computer program. Carrera's Unified Formulation (CUF) formulates axiomatic, displacement-based plate and shell models by postulating the through-thickness distribution of the displacement field within the weak form expressed by the Principle of Virtual Displacements (PVD). Following the systematic classification of Reddy [40], the displacement field can be described in either Equivalent Single Layer (ESL) or Layer-Wise (LW) manner. The ESL displacement field can be enhanced with Murakami's Zig-Zag Function (MZZF), which introduces the slope discontinuity at layers' interfaces, which is a necessary though not sufficient condition for satisfying the interlayer equilibrium [41,42]. Finally, the order of the polynomial approximation across the thickness of the panel (in an ESL description) or of the layer (in an LW description) may be freely selected, but it is taken to be the same for all displacement components. This latter limitation has been removed by the Generalized Unified Formulation (GUF) proposed by Demasi [43], where different orders can be introduced for the approximation of different displacement components. However, in both GUF and CUF the same hypotheses (i.e., ESL or LW description and approximation order) are used for all the plies of the composite. In order to overcome this limitation, a Sublaminate version of the GUF (S-GUF) has been recently proposed [44]. Following an idea early proposed by Reddy [45], the multilayered plate is subdivided into an arbitrary number of sublaminates, each constituted by an arbitrary number of physical plies, and the variable kinematics modeling expressed by GUF is employed independently in each sublaminate.

The objective of the present contribution is to propose benchmark solutions and an assessment of several displacement-based models for the global buckling and wrinkling instabilities of sandwich struts subjected to uniaxial compression loads. Ji and Waas $[30,31]$ provided reference solutions through an exact solution of the differential equations governing the 2D incremental elasticity problem formulated in a plane strain setting. Different models are formulated in the framework of the newly developed S-GUF, which allows a systematic investigation of the pertinence of the model assumptions. To this aim, the paper is organized as follows. Section 2 introduces the geometry of the considered problems and the notation employed throughout the paper. The governing equations and exact solution of the 2D incremental elasticity problem are described in Section 3. The approximate plate solution is formulated in Section 4, in which the S-GUF is introduced and extended to include the geometric stiffness matrix accounting for the initial stress. Section 5 presents the results of the numerical investigations and, finally, Section 6 summarizes concluding remarks and an outlook towards future research topics.

\section{Preliminaries}

We consider a sandwich strut, i.e., a wide beam in plane strain and subjected to an axial compressive load, generally composed of $N_{p}$ perfectly bonded plies of arbitrary thickness $h_{p}$. Attention is limited single-core, symmetric sandwich cross-sections that consist of a core of thickness $h_{c}$ that is perfectly bonded to the outer face sheets of thickness $h_{f}$ as illustrated in Fig. 1. Each face sheet is then

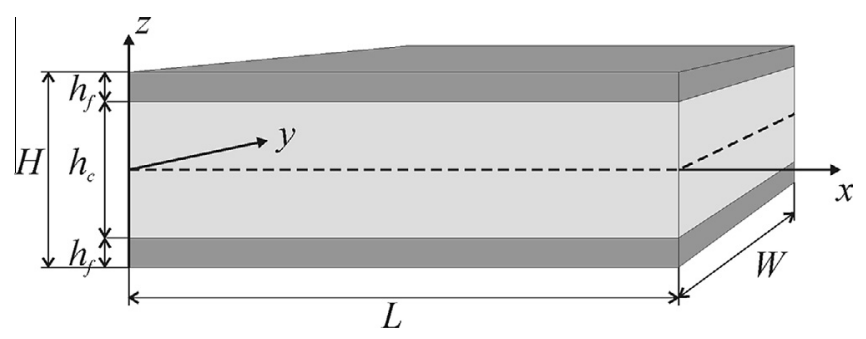

Fig. 1. Geometry of the symmetric sandwich plate.

composed of $N_{p}^{f}=\left(N_{p}-1\right) / 2$ of homogeneous, orthotropic and perfectly bonded plies. Cartesian coordinates $(x, y, z)=\left(x_{1}, x_{2}, x_{3}\right)$ are used to describe the undeformed strut occupying the volume $V=\Omega \times\left[-\frac{H}{2}, \frac{H}{2}\right]$, where $\Omega=[0, L] \times[0, W]$ is the mean surface that is taken to be coincident with the $x, y$ plane and $H$ is the constant total thickness of the sandwich strut.

The pre-buckled configuration of the strut is defined through an initial compressive axial strain along $x$ in the $x z$-plane, which can be thought of as generated by a shortening due to rigid end plates that are parallel to the $y z$-plane and move in the $x$-direction. As will be detailed later, the buckled configuration will be characterized by periodic modal shapes along the $x$-direction with a wavelength $2 L_{x}$. Fig. 2 illustrates the periodic antisymmetric and symmetric buckling modes that will be object of the present study. The global buckling mode corresponds to the antisymmetric mode with a half-wavelength $L_{x}=L$.

\section{Elasticity approach}

The bifurcation buckling problem is formulated by considering the adjacent equilibrium state the solid body arrives at upon a small perturbation of an initially stressed state. The incremental stresses $\sigma_{i j}^{*}$ must satisfy the general equilibrium equations

$\sigma_{i j, j}^{*}=0$

where Einstein's summation convention is employed with Latin indices $i, j, l, n=1,2,3$. The incremental Trefftz stress measure is employed, which is defined in terms of initial stress $\sigma_{i j}^{0}$ and perturbation stress $\sigma_{i j}^{\prime}$ according to

$\sigma_{i j}^{*}=\sigma_{i j}^{(2)}+\sigma_{l j}^{0} u_{i, l}$

This stress measure is work-conjugate to the Green-Lagrange finite strain tensor, which corresponds to the class of Doyle-Erickson strain tensors $\epsilon_{i j}^{(m)}$ with $m=2$ :

$\epsilon_{i j}^{(2)}=e_{i j}+\varepsilon_{i j} \quad$ with $\quad e_{i j}=\frac{1}{2}\left(u_{i_{, j}}+u_{j, i}\right) \quad$ and $\quad \varepsilon_{i j}=\frac{1}{2} u_{k, i} u_{k, j}$

Within this approach, the consistent constitutive model linking the perturbation stress and the finite strain employs constant stiffness moduli $\widetilde{C}_{i j l n}^{(2)}=C_{i j l n}$ :

$\sigma_{i j}^{\prime(2)}=\widetilde{C}_{i j l n} \epsilon_{l n}^{(2)}$

Note that the stiffness moduli are expressed in the Cartesian frame of the problem.

The sandwich strut is subjected to a uniform axial strain $\epsilon_{11}^{0}=\epsilon^{0}$, which produces in each ply $(p)$ a purely axial initial stress state $\sigma_{i j}^{0(p)}=\sigma_{11}^{0(p)}$ that depends on the elastic stiffness of the ply. The governing equilibrium equations are thus written for each ply of the sandwich strut as follows 

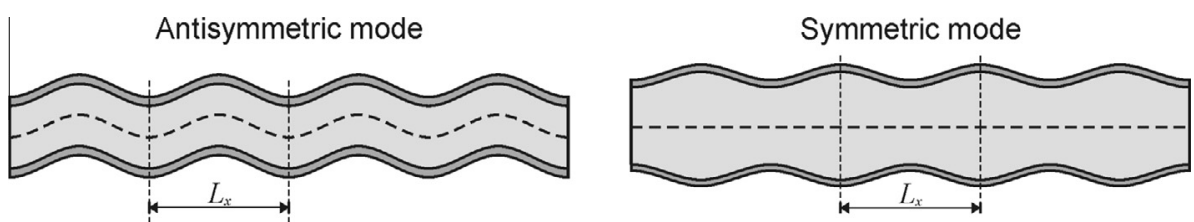

Fig. 2. Antisymmetric and symmetric periodic buckling modes.

$$
\begin{aligned}
& \frac{\partial}{\partial x_{1}}\left[\sigma_{11}^{\prime}+\sigma_{11}^{0(p)} u_{1,1}\right]+\frac{\partial \sigma_{12}^{\prime}}{\partial x_{2}}+\frac{\partial \sigma_{13}^{\prime}}{\partial x_{3}}=0 \\
& \frac{\partial}{\partial x_{1}}\left[\sigma_{12}^{\prime}+\sigma_{11}^{0(p)} u_{2,1}\right]+\frac{\partial \sigma_{22}^{\prime}}{\partial x_{2}}+\frac{\partial \sigma_{23}^{\prime}}{\partial x_{3}}=0 \\
& \frac{\partial}{\partial x_{1}}\left[\sigma_{13}^{\prime}+\sigma_{11}^{0(p)} u_{3,1}\right]+\frac{\partial \sigma_{23}^{\prime}}{\partial x_{2}}+\frac{\partial \sigma_{33}^{\prime}}{\partial x_{3}}=0
\end{aligned}
$$

where the superscript ${ }^{(2)}$ has been omitted from the perturbation stress components.

The constitutive law Eq. (4) and the strain-displacement relation Eq. (3) are substituted into the equilibrium equations and a system of homogeneous partial differential equations (PDE) is obtained. The solution of the bifurcation buckling problem consists in seeking the critical value of the parameter $\epsilon^{0}$ for which the above set of homogeneous PDE admits a non-trivial displacement field. Note that in the considered plane strain configuration the second of Eq. (5) is automatically verified.

\subsection{Analytical solution}

The present work focuses on periodic buckling and a harmonic solution is accordingly assumed for each ply in the following form:

$u_{1}^{p}\left(x, z_{p}\right)=\psi\left(z_{p}\right) \cos \left(x / L_{x}\right) \quad u_{3}^{p}\left(x, z_{p}\right)=\phi\left(z_{p}\right) \sin \left(x / L_{x}\right)$

where $L_{x}$ is the half-wavelength of the periodic in-plane modal shape and the functions $\psi\left(z_{p}\right)$ and $\phi\left(z_{p}\right)$ define the modal shape across the ply thickness. These are found to be linear combinations of four hyperbolic trigonometric functions whose full expression can be found elsewhere $[13,33,30]$. The solution Eq. (6) exactly satisfies the field equations Eq. (5) and introduces 4 unknown constants for each ply to be determined by appropriate boundary conditions. For symmetric sandwich struts, as those considered in the present work, only the upper half of the sandwich crosssection shall be considered. The 8 arbitrary constants associated to the solution for the top face and the core must thus verify 4 displacement and traction continuity conditions at the face-core interface and 2 homogeneous traction conditions at the top surface. The last two conditions are provided by imposing the modal shape across the thickness to be either symmetric or antisymmetric $[13,33,30]$. A classical eigenvalue problem is finally formulated by setting to zero the determinant of the resulting set of 8 algebraic equations in the 8 unknown coefficients. This may be represented in the following implicit form:

$f\left(\epsilon_{c r}^{0}, \eta\right)=0$

where the critical (buckling) strain $\epsilon_{c r}^{0}=\lambda \epsilon^{0}$, defined by the scaling factor $\lambda$ of the initial axial strain $\epsilon_{11}^{0}$, is obtained in function of a nondimensional half-wavelength of the periodic in-plane buckling mode $\eta=L_{x} / h_{f}$, where $h_{f}$ is the thickness of the sandwich face.

\section{Approximate plate solutions for linearized bifurcation analysis}

Approximate solutions to the governing equations of the buckling problem are found by referring to weak forms obtained from variational equations. The variational statement corresponding to the linearized stability analysis of a body subjected to initial stresses as defined by Eqs. (1)-(4) can be found in the book by Washizu [46]. The critical load is given by the scalar parameter $\lambda$ that multiplies the initial stress state $\sigma_{i j}^{0}$ and that satisfies

$\iint_{\Omega} \int_{H} \delta e_{i j} C_{i j l n} e_{l n}+\left(\delta u_{l, j}\right) \lambda \sigma_{i j}^{0} u_{l, i} \mathrm{~d} z \mathrm{~d} x \mathrm{~d} y=0$

where $\delta$ denotes virtual variation. The associated Euler equations are indeed the three equilibrium equations Eq. (1), where the incremental stresses are those given in Eq. (2) and the subsidiary conditions are those expressed by Eqs. (3) and (4).

Ji and Waas [30,31] have detailed out a displacement-based Finite Element Method (FEM) based on the above weak form in the framework of the plane strain elasticity, see also [29]. The importance of using correct work-conjugate strain and stress definitions has been pointed out along with the error that commercial FEM software can produce due to an inconsistent incremental elasticity formulation. In the present work, the weak form Eq. (8) is used as a starting point for formulating 2D plate models. Following the axiomatic approach, approximations are introduced by postulating the distribution of the displacement field along the transverse $z$-direction. Unified Formulations propose a way to express variable kinematics plate models according to the following compact index notation $[47,43,48]$ :

$u_{i}(x, y, z)=\sum_{\alpha=0}^{N_{u_{i}}} F_{\alpha u_{i}}(z) \hat{u}_{i \alpha}(x, y)$

where $F_{\alpha u_{i}}(z)\left(\alpha=0,1 \ldots N_{u_{i}}\right)$ are known approximating functions. Details about the way this approximation is formulated in this work are given in the subsequent section dedicated to the Sublaminate Generalized Unified Formulation (S-GUF). For the periodic buckling problem of orthotropic sandwich structures investigated in this study, a harmonic in-plane solution is formulated according to Navier's method $[35,15]$ :

$\hat{u}_{1 \alpha}(x, y)=U_{1 \alpha} \cos \frac{m \pi x}{L} \sin \frac{n \pi y}{W}$

$\hat{u}_{2 \alpha}(x, y)=U_{2 \alpha} \sin \frac{m \pi x}{L} \cos \frac{n \pi y}{W}$

$\hat{u}_{3 \alpha}(x, y)=U_{3 \alpha} \sin \frac{m \pi x}{L} \sin \frac{n \pi y}{W}$

The integers $m, n$ denote the number of half-waves of the periodic response along $x$ and $y$, respectively, and define, hence, the halfwavelengths $L_{x}=L / m, L_{y}=W / n$. For a simply-supported plate, the only approximations are those introduced across the transverse $z$-direction, i.e., they concern only the sandwich plate kinematics, because the Navier-type solution verifies in a strong form the PDE in the $x, y$-plane as well as the boundary conditions at $x=0, L$ and $y=0, W$.

The assumed solution obtained from the combination of Eqs. (10) and (9) is substituted into the variational Eq. (8). The integrals over the thickness $H$ of the sandwich strut are evaluated, which yields the weak form of the 2D governing equations. Those terms whose virtual variation is differentiated with respect to the inplane variables $x, y$ are integrated-by-parts for obtaining the strong form of the $2 \mathrm{D}$ governing equations, which are exactly verified by 
the adopted Navier solution. The plane strain condition is enforced by setting to zero all partial derivatives with respect to $x_{2}=y$. The governing equation for the bifurcation buckling is finally expressed in matrix form by the following classical eigenvalue problem:

$\delta \mathbf{U}_{(m)}^{\top}\left[\mathbf{K}_{(m)}+\lambda \mathbf{K}_{G(m)}\right] \mathbf{U}_{(m)}=\mathbf{0}$.

where superscript ${ }^{\top}$ is the transposition operator. The matrices $\mathbf{K}_{(m)}$ and $\mathbf{K}_{G(m)}$ are the linear stiffness matrix for the perturbed configuration and the geometric stiffness matrix associated to the initial stress field $\sigma_{i j}^{0}$, respectively.

Solution of the eigenvalue problem: critical loads and buckling loads. The matrices in Eq. (11) are square, symmetric and real and have a size $N_{D O F}$ dictated by the model approximations introduced by Eq. (9). For a given half-wavelength $L_{x}=a / m$ the plane strain $(n=0)$ eigenvalue problem yields $N_{D O F}$ real eigenvalues $\lambda_{(m)}$. Each eigenvalue corresponds to a critical load $N_{c r}(m)$ and the corresponding eigenvector is the through-thickness distribution of the buckled configuration. The lowest critical load obtained for all possible half-wavelengths $L_{x}=L / m$ defines the buckling load $P$ of the plate and the corresponding half-wavelength $L_{x}^{*}$ :

$P=\min _{m}\left(N_{c r}(m)\right) \quad$ at $m=m^{*} ; \quad L_{x}^{*}=L_{x}(m *)=\frac{L}{m^{*}}$

\subsection{Approximations in S-GUF}

In order to cope with the different mechanical and geometric properties of core and face sheets, that may be composed of several plies, it may be useful to introduce different approximations for these three layers. The recently proposed Sublaminate version of the Generalized Unified Formulation (S-GUF) [44] will be employed for modeling the sandwich strut as an assembly of $N L=3$ sublaminates. If the whole sandwich is modeled as a single sublaminate, $N L=1$, the S-GUF simply reduces to the established GUF [43]. In S-GUF, the integral along the transverse $z$-coordinate appearing in the variational Eq. (8) is hence split in NL sublaminate contributions:

$$
\begin{aligned}
\int_{H} \delta e_{i j} C_{i j l n} e_{l n}+\left(\delta u_{l_{, j}}\right) \lambda \sigma_{i j}^{0} u_{l, i} \mathrm{~d} z= & \sum_{k=1}^{N L} \int_{h_{k}} \delta e_{i j}^{k} \widetilde{C}_{i j l n}^{(p)} e_{l n}^{k} \\
& +\left(\delta u_{l_{, j}}^{k}\right) \lambda \sigma_{i j}^{0(p)} u_{l, i}^{k} \mathrm{~d} z_{k}
\end{aligned}
$$

where the superscript $(p)$ highlights the dependence of the stiffness coefficients and the initial stress on the ply $p$ inside the sublaminate $k$. In each sublaminate $k \in\{1, N L\}$, variable plate kinematics models are introduced following the GUF compact notation first introduced by Demasi [43]:

$u_{i}(x, y, z)=\sum_{\alpha_{u_{i}}=0}^{N_{u_{i}}^{k}} F_{\alpha_{u_{i}}}(z) \hat{u}_{i \alpha_{u_{i}}}^{k}(x, y)$

where $N_{u_{i}}^{k}$ is the order of the approximation of the variable $u_{i}$ inside the $k$ th sublaminate, which is a free input choice. The essential difference between the present Sublaminate-GUF and the GUF proposed by Demasi [43] lies in the fact that the order of the approximation $N_{u_{i}}^{k}$ depends not only on the displacement component (subscript $u_{i}$ ) but also on the sublaminate $k$. This permits to formulate different approximations for different sublaminates. The formulation of the variable kinematic model follows exactly the same way outlined by Demasi [43], where it is recalled that now the resulting model is defined for the sublaminate only instead of for the whole plate.

Once the sublaminate models Eq. (14) introduced in Eq. (13), the corresponding sublaminate integrals are evaluated. The model for the whole sandwich plate is finally obtained as a Layer-Wise assembly of all sublaminate models. The continuity of the assumed displacement field is hence exactly fulfilled at the interfaces between adjacent sublaminates.

\subsection{Approximations for the sublaminate}

When the $k$ th sublaminate is composed of $N_{p}^{k}>1$ plies, the SGUF model may be constructed with an ESL or LW description for the variable $u_{i}^{k}$. Note that the choice of the description can be made independently for each of the three displacement components. In the ESL case, the approximating functions $F_{\alpha_{u_{i}}}(z)$ are expressed in terms of the sublaminate-specific coordinate $z_{k}$;

$u_{i}^{k}\left(x, y, z_{k}\right)=\sum_{\alpha_{u_{i}}=0}^{N_{u_{i}}^{k}} F_{\alpha_{u_{i}}}\left(z_{k}\right) \hat{u}_{i \alpha_{u_{i}}}^{k}(x, y)$

In the LW case, the approximation is introduced in each ply, i.e., ply-specific coordinate $z_{p}$ must be used instead of $z_{k}$ in Eq. (15):

$u_{i}^{p}\left(x, y, z_{p}\right)=\sum_{\alpha_{u_{i}}=0}^{N_{u_{i}}^{k}} F_{\alpha_{u_{i}}}\left(z_{p}\right) \hat{u}_{i \alpha_{u_{i}}}^{p}(x, y)$

The approximation for the sublaminate is finally obtained upon assembling all contributions of the $N_{p}^{k}$ plies:

$u_{i}^{k}\left(x, y, z_{k}\right)=\sum_{p=1}^{N_{p}^{k}} u_{i}^{p}\left(x, y, z_{p}\right)=\sum_{p=1}^{N_{p}^{k}}\left\{\sum_{\alpha_{u_{i}}=0}^{N_{u_{i}}^{k}} F_{\alpha_{u_{i}}}\left(z_{p}\right) \hat{u}_{i \alpha_{u_{i}}}^{p}(x, y)\right\}$

Note that in an LW description the expansion order of each unknown $N_{u_{i}}^{k}$ is the same for all $N_{p}^{k}$ plies of the sublaminate.

For both ESL and LW descriptions, the sublaminate integral in Eq. (13) has to be evaluated as an assembly of ply-specific integrals in order to account for the ply-dependent stiffness coefficient:

$$
\begin{aligned}
\int_{h_{k}} \delta e_{i j}^{k} \widetilde{C}_{i j l n}^{(p)} e_{l n}^{k}+\left(\delta u_{l, j}^{k}\right) \lambda \sigma_{i j}^{0 k} u_{l, i}^{k} \mathrm{~d} z_{k}= & \sum_{p=1}^{N_{p}^{k}} \int_{z_{p}^{b}}^{z_{p}^{t}} \delta e_{i j}^{k} \widetilde{C}_{i j l n}^{p} e_{l n}^{k} \\
& +\left(\delta u_{l, j}^{k}\right) \lambda \sigma_{i j}^{0 p} u_{l, i}^{k} \mathrm{~d} z_{k}
\end{aligned}
$$

The assembly procedure of the ply integrals to build the sublaminate integral depends on the ESL or LW description of the variable $u_{i}$ and can be found elsewhere [44].

\subsubsection{The approximating functions}

The approximating functions $F_{\alpha_{u_{i}}}$ are polynomial functions defined in the non-dimensional coordinate $\zeta_{k} \in[-1,1]$ of the $k$ th sublaminate as follows

$$
\begin{aligned}
\text { if } N_{u_{i}}^{k}=0: & F_{0}\left(\zeta_{k}\right)=1 \\
\text { if } N_{u_{i}}^{k} \geqslant 1: & F_{0}\left(\zeta_{k}\right)=\frac{1+\zeta_{k}}{2} ; \quad F_{1}\left(\zeta_{k}\right)=\frac{1-\zeta_{k}}{2} ; \\
& F_{r}\left(\zeta_{k}\right)=P_{r}\left(\zeta_{k}\right)-P_{r-2}\left(\zeta_{k}\right) \quad \text { for } r=2,3, \ldots N_{u_{i}}^{k}
\end{aligned}
$$

where $P_{r}\left(\zeta_{k}\right)$ is Legendre's polynomial of order $r$ that can be defined recursively from following relations [49]

$P_{0}\left(\zeta_{k}\right)=1 ; \quad P_{1}\left(\zeta_{k}\right)=\zeta_{k} ; \quad P_{r+1}\left(\zeta_{k}\right)=\frac{(2 r+1) \zeta_{k} P_{r}\left(\zeta_{k}\right)-r P_{r-1}\left(\zeta_{k}\right)}{r+1}$

These functions are used inside the $k$ th sublaminate irrespective of the ESL or LW descriptions of the variable $u_{i}$. The chosen approximating functions ease the imposition of the continuity at interfaces 
between sublaminates as well as between plies inside a sublaminate described in a LW manner. Note that if a Layer-Wise description is chosen inside the $k$ th sublaminate, the non-dimensional ply-specific coordinate $\zeta_{p}$ should be used instead of $\zeta_{k}$ in Eqs. (18) and (19). The relation between these non-dimensional coordinates reads

$\zeta_{p}=\frac{h_{k}}{h_{p}} \zeta_{k}+\frac{2}{h_{p}}\left(z_{0 k}-z_{0 p}\right)$

where $z_{0 k}$ and $z_{0 p}$ are the $z$-coordinates of the mean surfaces of the $k$ th layer and the $p$ th ply, respectively.

\subsubsection{Generic kernel for the linear stiffness matrix}

The proposed S-GUF maintains the main feature of Unified Formulations, which consists in expressing variable kinematics models in terms of kernels or fundamental nuclei that are invariant with respect to the chosen model approximations. The kernels for the linear stiffness matrix $\mathbf{K}$ of Eq. (11) are obtained from the virtual variation of internal strain energy $\delta \Pi_{\text {int }}$ during the perturbation from the initially stressed state:

$\delta \Pi_{i n t}=\delta \mathbf{U}_{(m, n)}^{\top} \mathbf{K}_{(m, n)} \mathbf{U}_{(m, n)}=\iint_{\Omega} \int_{H} \delta e_{i j} C_{i j l n} e_{l n} \mathrm{~d} z \mathrm{~d} x \mathrm{~d} y$

Introducing the S-GUF expansion Eq. (14) for the variables $u_{i}$ and $u_{j}$, the virtual variation of internal strain energy can be split into sublaminate contributions according to Eq. (13):

$\delta \Pi_{\text {int }}=\sum_{k=1}^{N L} \delta \Pi_{i n t}^{k}=\sum_{k=1}^{N L} \delta u_{i \alpha_{u_{i}}}^{k}\left(K_{u_{i} u_{j}}^{k \alpha_{u_{j}} \beta_{u_{j}}}\right) u_{j \beta_{u_{j}}}^{k}$

A generic expression for all terms contributing to the $k$ th sublaminate can be then written from Eq. (17) in the following way:

$$
\begin{aligned}
\delta u_{i \alpha_{u_{i}}}^{k}\left(K_{u_{i} u_{j} Q S}^{k \alpha_{u_{i}} \beta_{u_{j}}}\right) u_{j \beta_{u_{j}}}^{k}= & \iint_{\Omega}\left(\partial_{(x, y)}^{(i)} \delta \hat{u}_{i \alpha_{u_{i}}}^{k}(x, y)\right)\left\{\sum_{p=1}^{N_{p}^{k}} \widetilde{Z}_{u_{i} u_{j} Q S}^{p \alpha_{u_{i}} \beta_{u_{j}}}\right\} \\
& \times\left(\partial_{(x, y)}^{(j)} \hat{u}_{j \beta_{u_{j}}}^{k}(x, y)\right) \mathrm{d} x \mathrm{~d} y
\end{aligned}
$$

where, depending on the considered term, the displacement component $u_{i}$ or $u_{j}$ may be derived with respect to the in-plane coordinates, which is indicated by the notation $\partial_{(x, y)}^{(i, j)}$. The ply-integral $\widetilde{Z}_{u_{i} u_{j} Q S}^{p \alpha_{u_{i}} \beta_{u_{j}}}$ is defined as

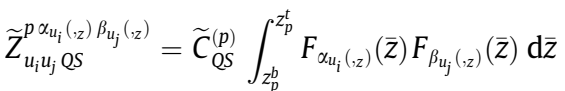

with $\bar{z}=z_{k}$ for an ESL description and $\bar{z}=z_{p}$ for a LW description. Depending on the considered term, the displacement components $u_{i}$ or $u_{j}$ may be derived with respect to the transverse coordinate $z$, which is indicated by the subscript $\left.{ }_{, z}\right)$. The conventional Voigt notation has been introduced in order to write the constitutive Eq. (4) in matrix form as

$\sigma_{Q}=\widetilde{C}_{Q S} e_{S} \quad(Q, S=1,2, \ldots 6)$

with

$e_{1}=e_{11} ; \quad e_{2}=e_{22} ; \quad e_{3}=e_{33} ; \quad e_{4}=2 e_{23} ; \quad e_{5}=2 e_{13} ;$

$e_{6}=2 e_{12}$;

$\sigma_{1}=\sigma_{11}^{\prime} ; \quad \sigma_{2}=\sigma_{22}^{\prime} ; \quad \sigma_{3}=\sigma_{33}^{\prime} ; \quad \sigma_{4}=\sigma_{23}^{\prime} ; \quad \sigma_{5}=\sigma_{13}^{\prime} ;$

$\sigma_{6}=\sigma_{12}^{\prime}$

More details can be found in [44] and a full derivation of all kernels can be found in the detailed paper by Demasi [43]. All ply-integrals $\widetilde{Z}_{u_{i} u_{j} Q S}^{p \alpha_{u_{i}(, z)} \beta_{u_{j}}(, z)}$ as well as the kernels $K_{u_{i} u_{j}}^{p \alpha_{u_{i}} \beta_{u_{j}}}$ for the present Navier-type solution are reported in Appendix A.

\subsubsection{Kernel for the geometric stiffness matrix}

This work considers a uniaxial stress $\sigma_{11}^{0(p)}$ acting in each ply that is generated by a uniform strain $\epsilon_{11}^{0}$ induced by an end shortening of the whole sandwich strut within the plane strain setting. The uniaxial initial stress is hence defined from the combination of the condition $\sigma_{33}^{0(p)}=0$ and of the plane strain condition $\epsilon_{22}^{0}=0$ [50], which yields

$\sigma_{11}^{0(p)}=Q_{11}^{(p)} \epsilon_{11}^{0} \quad$ with $\quad Q_{11}^{(p)}=\widetilde{C}_{11}^{(p)}-\frac{\widetilde{C}_{13}^{(p)} \widetilde{C}_{31}^{(p)}}{\widetilde{C}_{33}^{(p)}}$

It appears useful to state the equivalence between this definition and that used in [30], where the initial stress in each ply is defined in terms of the total compressive load $P$ as

$\sigma_{11}^{0(p)}=\frac{Q_{11}^{(p)}}{A_{11} W} P$

For a composite plate composed of $N_{p}$ plies, the axial membrane stiffness $A_{11}$ is

$A_{11}=\sum_{p=1}^{N_{p}} Q_{11}^{(p)} h_{p}$

with $Q_{11}^{(p)}=E^{p} /\left(1-\left(v^{p}\right)^{2}\right)$ for isotropic materials. Introducing an initial uniform stress $\Sigma^{0}=P /(H W)$ the initial stresses in each ply can be hence expressed as

$\sigma_{11}^{0(p)}=\frac{Q_{11}^{(p)} H}{A_{11}} \Sigma^{0}$

which is equivalent to Eq. (25) once the uniform initial strain is identified as

$\epsilon_{11}^{0}=\frac{H}{A_{11}} \Sigma^{0}=\frac{P}{A_{11} W}$

The kernels for the geometric stiffness matrix are identified from the ply contribution to the virtual work done by the initial stress. Introducing the definition of the initial stress field Eq. (25) into the variational Eq. (17) one has

$$
\begin{aligned}
\delta \mathbf{U}_{(m, n)}^{\top} \lambda \mathbf{K}_{G(m, n)} \mathbf{U}_{(m, n)} & =\iint_{\Omega} \int_{H} Q_{11}^{(p)} \lambda \epsilon_{11}^{0}\left(u_{1,1} \delta u_{1,1}+u_{2,1} \delta u_{2,1}+u_{3,1} \delta u_{3,1}\right) \mathrm{d} z \mathrm{~d} x \mathrm{~d} y
\end{aligned}
$$

If von Kàrmàn approximation is used for the non-linear strain definition, the membrane contributions are discarded, i.e., $u_{1,1}=u_{2,1}=0$ is set.

Introducing the S-GUF approximation (14), the following expression is obtained for the contribution of the $k$ th sublaminate:

$\delta u_{i \alpha_{u_{i}}}^{k}\left(\lambda K_{G u_{i} u_{i} 11}^{k \alpha_{u_{i}} \beta_{u_{i}}}\right) u_{i \beta_{u_{i}}}^{k}=\lambda \epsilon_{11}^{0} \iint_{\Omega} \frac{\partial \delta \hat{u}_{i \alpha_{u_{i}}}(x, y)}{\partial x_{1}}\left\{\sum_{p=1}^{N_{p}^{k}} \widetilde{Z}_{E u_{i} u_{i} 11}^{p \alpha_{u_{i}} \beta_{u_{i}}}\right\} \frac{\partial \hat{u}_{i \beta_{u_{i}}}(x, y)}{\partial x_{1}}$

with the ply-integral defined as

$$
\widetilde{Z}_{E u_{i} u_{i} 11}^{p \alpha_{u_{i}} \beta_{u_{i}}}=Q_{11}^{(p)} \int_{z_{p}^{b}}^{z_{p}^{t}} F_{\alpha_{u_{i}}}(\bar{z}) F_{\beta_{u_{i}}}(\bar{z}) \mathrm{d} \bar{z}
$$

The ply-integral $\widetilde{Z}_{E u_{i} u_{i} 11}^{p \alpha_{u_{i}} \beta_{u_{i}}}$ is the kernel of the geometric stiffness matrix for the considered initial load defined in terms of uniform strain. Its expression is invariant with respect to the expansion order used for the displacement $u_{i}^{k}$, the ESL or LW description of $u_{i}^{k}$ inside the sublaminate and the solution adopted in $x y$-plane. The kernels for the geometric stiffness matrix $K_{G u_{i} u_{i}}^{p \alpha_{u_{i}} \beta_{u_{i}}}$ for the considered Navier-type solution are explicitly reported in Appendix A. 


\subsubsection{Size of the resulting arrays}

The sublaminate kernels $K_{u_{i} u_{j}}^{p \alpha_{u_{i}} \beta_{u_{j}}}$ and $K_{G u_{i} u_{i}}^{k \alpha_{u_{i}} \beta_{u_{i}}}$ are first expanded over the indices $\alpha_{u_{i}}=0,1, \ldots N_{u_{i}}^{k}$ and $\beta_{u_{j}}=0,1, \ldots N_{u_{j}}^{k}$, which yields the ply contribution to $k$ th sublaminate as a $\left(1+N_{u_{i}}^{k}\right) \times\left(1+N_{u_{j}}^{k}\right)$ array. The assembly over all plies inside the sublaminate is then performed as formally described by Eq. (17). The resulting size of the arrays depends on whether the $u_{i}^{k}$ and $u_{j}^{k}$ variables are described in ESL or LW manner, as given in Table 1.

\section{Numerical results}

Numerical tests are performed for the periodic buckling of symmetric sandwich plates subjected to a uniaxial uniform strain as described in the previous Sections. Several 2D plate models are assessed towards the elasticity solutions in the 2D plane strain setting. The S-GUF is here used to represent and assess several classical models proposed in open literature for the analysis of buckling of sandwich panels. In the following, the models considered in the assessment and their representation within S-GUF are discussed. Subsequently, numerical results are presented for sandwich panels with orthotropic and isotropic core.

\subsection{Considered sandwich plate models}

This section introduces briefly the axiomatic models considered in the numerical assessment. A class of models originally developed for sandwich panels with honeycomb core considers the in-plane stiffness of the core negligible compared to the transverse one. The models proposed in $[9,51,11]$ refer to this anti-plane stress assumption and formulate a core kinematics with cubic and quadratic expansions for the in-plane and transverse displacements, respectively. In the following, these models will be referred to as HSAPT. Besides the classical HSAPT model, in which the axial Young's modulus of the core is set to zero, a modified version of this class of models, denoted $\mathrm{HSAPT}_{\sigma^{0}}$, is discussed in this work: in HSAPT $_{\sigma^{0}}$ the axial stiffness of the core is retained in the definition of the perturbation stresses Eq. (4), but it does not contribute to the initial stress, i.e., the core axial stiffness is neglected only in the geometric stiffness matrix. In both the HSAPT and HSAPT $\sigma^{0}$ models, the initial stress is entirely carried by the face sheets. Note that in this configuration the dominant buckling mode can be a non-periodic short wavelength one, known as edge buckling [33]. Phan et al. [52,53] analyzed sandwich buckling with an enhanced version of HSAPT, which includes the in-plane stiffness of the core and will be referred to as EHSAPT model. Hohe and Librescu [54] proposed a sandwich plate model based on a core kinematics with quadratic and linear expansions for the in-plane and transverse displacements, respectively. This model will be denoted $\mathrm{HL}$ in the following and, in comparison to HSAPT, represents the core with a lower-order kinematics but it retains its in-plane stiffness. While all models discussed so far adopt the Euler-Bernoulli-Kirchhoff hypothesis that discards the transverse deformation in the face

Table 1

Array size for a sublaminate constituted of $N_{p}^{k}$ plies and with expansion orders $N_{u_{i}}^{k}$ and $N_{u_{j}}^{k}$

\begin{tabular}{lll}
\hline Description for $u_{i}^{k}$ & Description for $u_{j}^{k}$ & Size $\left(K_{u_{i} u_{j}}^{k}\right)=\operatorname{size}\left(Z_{u_{i} u_{j} Q S}^{k}\right)$ \\
\hline ESL & ESL & $\left(1+N_{u_{i}}^{k}\right) \times\left(1+N_{u_{j}}^{k}\right)$ \\
ESL & LW & $\left(1+N_{u_{i}}^{k}\right) \times\left(N_{p}^{k} N_{u_{j}}^{k}-1\right)$ \\
LW & ESL & $\left(N_{p}^{k} N_{u_{i}}^{k}-1\right) \times\left(1+N_{u_{j}}^{k}\right)$ \\
LW & LW & $\left(N_{p}^{k} N_{u_{i}}^{k}-1\right) \times\left(N_{p}^{k} N_{u_{j}}^{k}-1\right)$ \\
\hline
\end{tabular}

Table 2

S-GUF representation of the models for sandwich panels considered in the numerical assessment.

\begin{tabular}{lllll}
\hline Acronym & Face model & Core kinematics & Core assumptions & NDOF \\
\hline HL & CLT & $N_{u_{1}}=2, N_{u_{3}}=1$ & & 12 \\
DY & FSDT & $N_{u_{1}}=2, N_{u_{3}}=1$ & & 12 \\
HSAPT & CLT & $N_{u_{1}}=3, N_{u_{3}}=2$ & $E_{11}^{c}=0 ; \sigma_{11}^{O(c)}=0$ & 15 \\
HSAPT $_{\sigma^{0}}$ & CLT & $N_{u_{1}}=3, N_{u_{3}}=2$ & $\sigma_{11}^{0(c)}=0$ & 15 \\
EHSAPT & CLT & $N_{u_{1}}=3, N_{u_{3}}=2$ & & 15 \\
\hline
\end{tabular}

sheets (CLT model), the DY model proposed by Dawe and Yuan [55] employs the same core kinematics of the HL model but includes a constant transverse shear strain in the face sheets by referring to an FSDT model.

The S-GUF allows to model the sandwich plate in terms of three-layer models with different kinematics for the three sublaminates representing the face sheets and the core. Since S-GUF consists of a variable kinematics approach, all previously discussed HL, DY, HSAPT, HSAPT $\sigma_{\sigma^{0}}$ and EHSAPT kinematics are particular cases of S-GUF models and can thus be represented within the same software with the model definition summarized in Table 2. Following the standard nomenclature for Unified Formulation models, capital 'E' and 'L' denote ESL and LW descriptions, respectively; capital ' $D$ ' indicates the displacement-based approach; the order of the polynomial expansions for $u_{1}$ and $u_{3}$ is specified in the subscript. Therefore, the classical CLT and FSDT models are both represented in S-GUF notation as $\mathrm{ED}_{10}$, where a 0th-order implies a constant approximation, see Eq. (18a). Note that the NDOF indicated in Table 2 is referred to the S-GUF representation. CLT and FSDT have thus the same NDOF, where the infinite transverse shear rigidity of CLT is included via a penalty factor whereas a unit shear correction factor is used for FSDT. It should be finally pointed out that several models in the literature have been proposed for isotropic core materials only as, e.g., [9,11], but this limitation has not been retained in the present S-GUF representation in which all materials are considered as orthotropic.

\subsection{Sandwich with orthotropic core}

The case study proposed by Ji and Waas [31] is considered in order to validate the S-GUF buckling analysis. A short sandwich strut with $H=1$ [mm], $L=3 H$ is made of two identical face sheets of thickness $h_{f}=0.1[\mathrm{~mm}]$ and a core of thickness $h_{c}=0.8[\mathrm{~mm}]$. Two material configurations are considered: an orthotropic core is combined with an isotropic face (Case 1) and an orthotropic core is combined with an orthotropic face (Case 2). The complete set of material data used for Case 1 and Case 2 are reported in Table 3 and Table 4, respectively. The results are given in Fig. 3 and report the critical loads for the global buckling mode $\left(L_{x}=L\right)$. The computed buckling loads $P$ are normalized with respect to the global beam buckling load defined as

$P_{G}=\frac{P_{E}}{1+\left(P_{E} / \overline{G A}\right)}$

Table 3

Material data for Case 1 with isotropic face sheets and orthotropic core.

\begin{tabular}{lll}
\hline Case 1 & Property & Value \\
\hline Face sheets & $E^{f}[\mathrm{GPa}]$ & 30 \\
& $v^{f}$ & 0.3 \\
Core & $E_{33}^{c}[\mathrm{GPa}]$ & $4 \times G_{13}^{c}$ \\
& $G_{13}^{c}[\mathrm{GPa}]$ & $G^{f} / 1000$ \\
& $v_{13}^{c}$ & 0.25
\end{tabular}


Table 4

Material data for Case 2 with orthotropic face sheets and core.

\begin{tabular}{lll}
\hline Case 2 & Property & Value \\
\hline Face sheets & $E_{11}^{f}[\mathrm{GPa}]$ & 107 \\
& $E_{33}^{f}[\mathrm{GPa}]$ & 15 \\
& $G_{13}^{f}[\mathrm{GPa}]$ & 4.3 \\
& $v_{13}^{f}$ & 0.3 \\
Core & $E_{33}^{c}[\mathrm{GPa}]$ & $4 \times G_{13}^{c}$ \\
& $G_{13}^{c}[\mathrm{GPa}]$ & $G_{13}^{f} / 200$ \\
& $v_{13}^{c}$ & 0.25 \\
\hline
\end{tabular}

where $P_{E}$ is the conventional Euler load

$P_{E}=\frac{\pi^{2} \overline{E I}}{L^{2}} \quad$ with $\overline{E I}=W\left[E^{f} \frac{h_{f}^{3}}{6}+E^{f} h_{f} \frac{\left(h_{f}+h_{c}\right)^{2}}{2}+E^{c} \frac{h_{c}^{3}}{12}\right]$

and $\overline{G A}$ is the shear stiffness defined by Huang and Kardomateas [56] as

$$
\begin{aligned}
\overline{G A}= & \frac{1}{2 W}\left[\frac{E^{f^{2}}}{4 \overline{E I^{2} G^{f}}}\left(e^{4} h_{f}-\frac{2}{3} e^{2}\left(e^{3}-d^{3}\right)+\frac{1}{5}\left(e^{5}-d^{5}\right)\right)\right. \\
& \left.\left.+\frac{E^{f^{2}}}{\overline{E I^{2} G^{c}}} h_{c}^{2} c^{2} d+\frac{2}{15} \frac{E^{c 2}}{E^{f^{2}}} d^{5}+\frac{2}{3} \frac{E^{c}}{E^{f}} h_{f} c d^{3}\right)\right]^{-1}
\end{aligned}
$$

with $e=h_{f}+h_{c} / 2, c=\left(h_{f}+h_{c}\right) / 2$ and $d=h_{c} / 2$, see also [57].

The evolution of the normalized buckling load with respect to the core orthotropy $E_{11}^{c} / E_{22}^{c}$ is reported in Fig. 3(a) for both Case 1 and Case 2. The approximated plate results are obtained with the high-order S-GUF model $\mathrm{ED}_{4,4} / \mathrm{ED}_{8,8}$ (fourth-order expansion for the faces and eigth-order expansion for the core). An excellent agreement is shown between the S-GUF model and the reference FE solution of [31]. Fig. 3(b) compares the non-dimensional buckling loads obtained for varying aspect ratio $L / H$ of the sandwich strut. The total thickness of the sandwich has been kept constant with $h_{f}=0.1$ and $h_{c}=0.8[\mathrm{~mm}]$. Both Case 1 and Case 2 are again considered: the results in Fig. 3(b) refer to an axial Young's modulus that corresponds to $E_{11}^{c}=400 \times G_{13}^{c}$ for Case 2 and to $E_{11}^{c}=480 \times G_{13}^{c}$ for Case 2 . The agreement between the S-GUF plate model and the reference solution obtained by the accurate FE computations of [31] is very good. The small discrepancies occurring for the extremely short strut $(L / H=2)$ are attributed to the slightly different boundary conditions that have been used in the FE computation with respect to the simply-support conditions implied by Navier's solution. Note that, since the edge conditions of the FE simulation $-u_{1}(x=0, z)=u_{1}(x=L, z)=0$ and $u_{3}(x=0, z=0)=u_{3}(x=L, z=0)=0$ - are more similar to a clamp [31], the beam length $L=3 H$ used for the Navier solution is one half of that used in [31].

The assessment of the models reported in Table 2 for the varying orthotropic ratio $E_{11}^{c} / E_{33}^{c}$ is illustrated in Fig. 4(a) for Case 1 and Fig. 4(b) for Case 2. The non-dimensional buckling load predicted by the HSAPT model is clearly insensitive with respect to the $E_{11}^{c} / E_{33}^{c}$ ratio: its value is $P / P_{G}=0.88$, which is a conservative estimate, and is not reported in Fig. 4. On the contrary, the HSAPT $\sigma^{0}$ model does systematically provide a largely non-conservative prediction of the buckling load. The errors increase with the core axial stiffness and can be as high as $70 \%$ for Case 1 (isotropic faces) and $40 \%$ for Case 2 (orthotropic faces). The lower-order models HL and DY behave similarly but with smaller error magnitudes. Comparing the HL and DY results in Fig. 4, one notices that the assumption of zero transverse shear deformability of the face sheets appears to be irrelevant for the isotropic faces (Case 1 ) but produces a certain inaccuracy when the faces are orthotropic (Case 2). The error introduced by this hypothesis is also shown to slightly grow with the core stiffness. Moreover, from Fig. 4(b) it appears that this assumption is the main source of error of the high-order EHSAPT model.

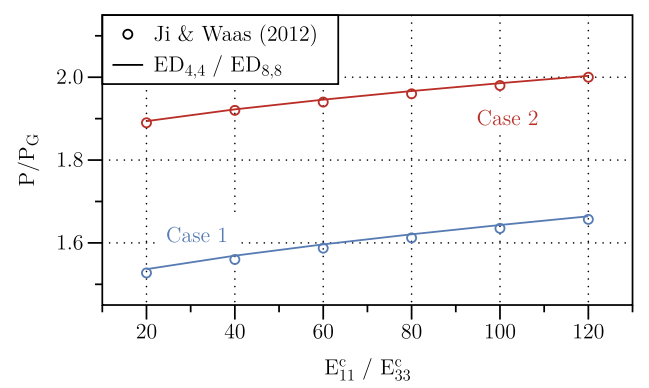

(a) Global buckling vs orthotropy ratio

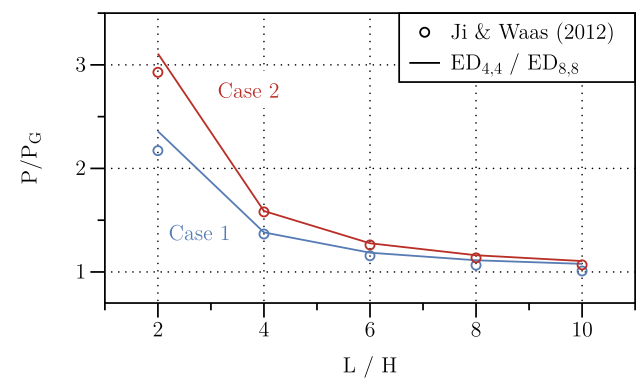

(b) Global buckling $v s$ aspect ratio

Fig. 3. Evolution of normalized global buckling load with core othotropy and aspect ratio.

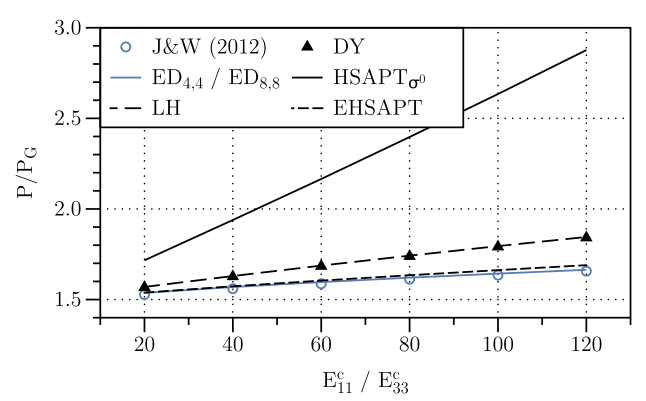

(a) Case 1 (isotropic face sheets)

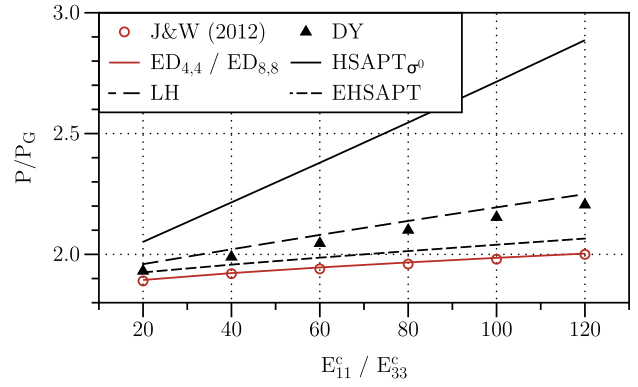

(b) Case 2 (orthotropic face sheets)

Fig. 4. Model assessment with respect to core othotropy. 


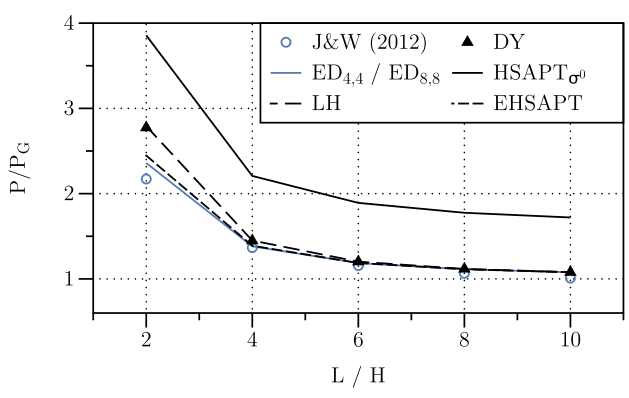

(a) Case 1 (isotropic face sheets)

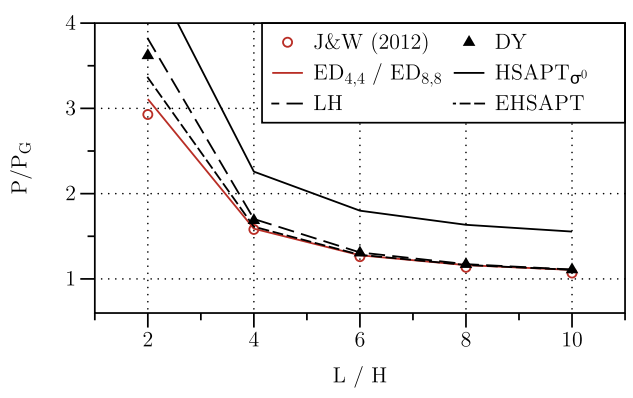

(b) Case 2 (orthotropic face sheets)

Fig. 5. Model assessment with respect to aspect ratio.

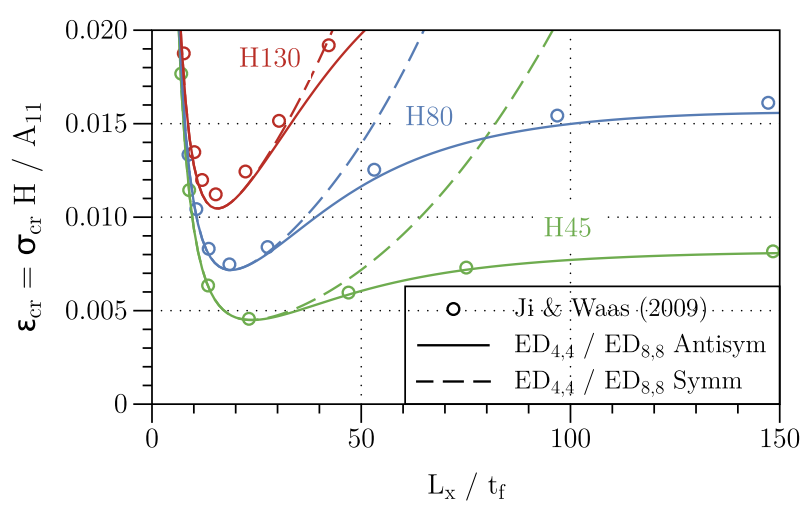

Fig. 6. Fagerberg case study: critical strain $v s$ buckling wavelength for three different core qualities.

Fig. 5 reports the model assessment for the varying aspect ratio $L / H$ and similar conclusions can be drawn.

\subsection{Sandwich with isotropic core}

The symmetric sandwich plate experimentally investigated by Fagerberg [5] is considered in the plane strain setting and with a model consisting of three layers of isotropic materials [30]. The geometry of the strut is defined by $L=200[\mathrm{~mm}], H=70[\mathrm{~mm}]$ with a core of thickness $h_{c}=68[\mathrm{~mm}]$ and two faces of thickness $h_{f}=1[\mathrm{~mm}]$ each. The faces are four-ply $[0 / 90]_{s}$ laminates, where each ply has equal thickness $h_{p}=h_{f} / 4=0.25[\mathrm{~mm}]$ and following material data (moduli in [GPa]): $E_{11}^{f}=107, E_{22}^{f}=15, G_{12}^{f}=4.3$, major Poisson's ratio $v_{12}^{f}=0.3[5,30]$. The equivalent axial Young's modulus and Poisson's ratio for the faces are obtained through the membrane stiffness matrix of the CLT: the isotropic data used in the study are thus $E_{x}^{f}=61.443[\mathrm{GPa}]$ and $v_{x y}^{f}=v_{x z}^{f}=0.0738$. The core, made out of Divinycell $\mathrm{H}$-grade materials, is modeled as an isotropic material with $v_{c}=0.3$ and Young's modulus $E_{c}$ depending on the foam quality $[5,30]: E_{c}=40[\mathrm{MPa}]$ for an $\mathrm{H} 45$ foam, $E_{c}=80[\mathrm{MPa}]$ for an $\mathrm{H} 80$ foam and $E_{c}=140[\mathrm{MPa}$ ] for an $\mathrm{H} 130$ foam.

Fig. 6 reports the critical strain $\epsilon_{c r}=\sigma_{c r} H / A_{11}$ in function of the normalized half-wavelength $L_{x} / h_{f}$. The results obtained with the high-order S-GUF model $\mathrm{ED}_{4,4} / \mathrm{ED}_{8,8}$ compare well against the analytical elasticity solution extracted from Ji and Waas [30]. Both symmetric and antisymmetric wrinkling modes are considered and it is apparent that for the considered isotropic cores the dominant buckling mode is the antisymmetric one, see also [15]. In the following, only the anti-symmetric wrinkling mode shall thus be addressed.

An assessment of several three-layers sandwich models is proposed in Fig. 7 for the configurations with $\mathrm{H} 45$ (weak core) and H130 (stiff core) foams. For this assessment, the face sheets are modeled as laminated plates, i.e., the complete set of orthotropic material properties of each ply is used, while the core is still modeled as an isotropic layer. The reference solution is here taken to be the $\mathrm{LD}_{4,4} / \mathrm{ED}_{8,8}$ model, in which each ply of the laminated faces has a fourth-expansion for both $u_{1}$ and $u_{3}$. Results in Fig. 7 are reported in terms of normalized buckling loads $P / P_{G}$, where $P_{G}$ is the global beam buckling load defined in Eq. (29) and for which the following face moduli are used (values in [GPa]): $E^{f}=61.443, G^{f}=4.3$. The assessment in Fig. 7(a) indicates that the HL and DY models are unable to grasp the antisymmetric wrinkling of the sandwich panel. An at least linear variation of the transverse normal strain is required for correctly capturing this short wavelength response. The HSAPT model is seen to underestimate the critical loads over the whole wavelength range. For the weak H45 core, Fig. 7(a) shows that $\mathrm{HSAPT}_{\sigma^{0}}$ provides results with similar accuracy as EHSAPT, i.e., the core does effectively not carry much of the initial load. However, for the stiffer $\mathrm{H} 130$ core, it is interesting to observe that HSAPT $_{\sigma^{0}}$ and EHSAPT yield similar accuracy only in the short wavelength (wrinkling) regime; higher global buckling load

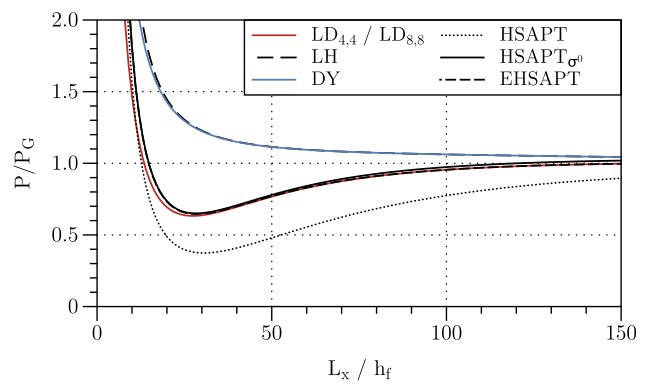

(a) Weak $\mathrm{H} 45$ core

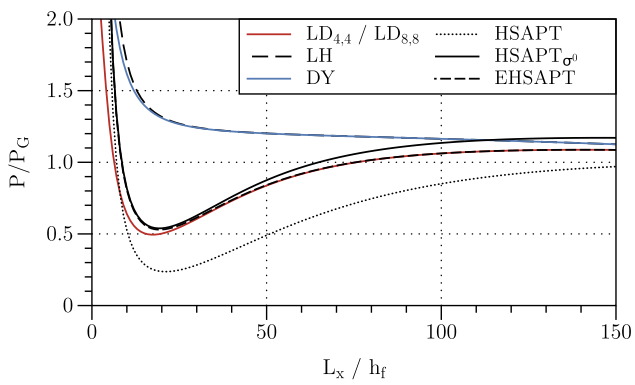

(b) Stiff H130 core

Fig. 7. Model assessment for the Fagerberg case study. 
(long wavelength regime) is predicted by the $\mathrm{HSAPT}_{\sigma^{0}}$ model, see Fig. 7(b).

\section{Conclusions and outlook}

This paper proposed an assessment of several well known sandwich plate models for the global and local buckling of struts in the plane strain setting. Benchmark solutions are provided by analytical solutions and accurate FE computations of the elasticity equations. All assessed plate models are formulated in the framework of a recently proposed Sublaminate Generalized Unified Formulation, a variable kinematics approach in which different approximations can be introduced in the face sheets and the core independently. The presented numerical results concern short sandwich struts subjected to a uniform compressive strain and whose face sheets and core consist of isotropic and orthotropic homogeneous materials. High-order approximations are shown to well recover the reference solutions. Based on the considered case studies, the following conclusions can be drawn from the proposed assessment:

- an at least quadratic approximation is required for the transverse displacement in the core in order to grasp the local buckling instability;

- if the axial stiffness of the core is neglected, lower (conservative) buckling loads are usually obtained;

- if the initial load is carried by the skins only, the predicted critical load for periodic buckling is usually not conservative;

- the EHSAPT model provides satisfactory accuracy, but the CLT approximation in the face sheets can produce a higher critical load.

The authors dedicate this work to the lifelong contributions by Prof. J. N. Reddy to the literature dealing with the mechanics of composite plates and shells.

\section{Appendix A. GUF kernels}

The instantiations of the generic kernel $Z_{u_{i} u_{j} P Q}^{p \alpha_{u_{i}} \beta_{u_{j}}}$ for the linear stiffness matrix are the following:

$$
\begin{aligned}
& \left\{Z_{u_{1} u_{1} 11}^{p \alpha_{u_{1}} \beta_{u_{1}}}, Z_{u_{1} u_{1} 66}^{p \alpha_{u_{1}} \beta_{u_{1}}}\right\}=\left\{\widetilde{C}_{11}^{(p)}, \widetilde{C}_{66}^{(p)}\right\} \int_{z_{p}^{b}}^{z_{p}^{t}} F_{\alpha_{u_{1}}}\left(z_{k}\right) F_{\beta_{u_{1}}}\left(z_{k}\right) \mathrm{d} z_{k} ; \\
& Z_{u_{1} u_{1} 55}^{p \alpha_{u_{1} \beta_{u_{1}}}}=\widetilde{C}_{55}^{(p)} \int_{z_{p}^{b}}^{z_{p}^{t}} \frac{\partial F_{\alpha_{u_{1}}}\left(z_{k}\right)}{\partial z} \frac{\partial F_{\beta_{u_{1}}}\left(z_{k}\right)}{\partial z} \mathrm{~d} z_{k} ; \\
& \left\{Z_{u_{1} u_{2} 12}^{p \alpha_{u_{1} \beta_{u_{2}}}}, Z_{u_{1} u_{2} 66}^{p \alpha_{u_{1}} \beta_{u_{2}}}\right\}=\left\{\widetilde{C}_{12}^{(p)}, \widetilde{C}_{66}^{(p)}\right\} \int_{z_{p}^{b}}^{z_{p}^{t}} F_{\alpha_{u_{1}}}\left(z_{k}\right) F_{\beta_{u_{2}}}\left(z_{k}\right) \mathrm{d} z_{k} ; \\
& Z_{u_{1} u_{3} 13}^{p \alpha_{u_{1} \beta_{u_{3}}}}=\widetilde{C}_{13}^{(p)} \int_{z_{p}^{b}}^{z_{p}^{t}} F_{\alpha_{u_{1}}}\left(z_{k}\right) \frac{\partial F_{\beta_{u_{3}}}\left(z_{k}\right)}{\partial z} \mathrm{~d} z_{k} ; \\
& Z_{u_{1} u_{3} 55}^{p \alpha_{u_{1}} \beta_{u_{3}}}=\widetilde{C}_{55}^{(p)} \int_{z_{p}^{b}}^{z_{p}^{t}} \frac{\partial F_{\alpha_{u_{1}}}\left(z_{k}\right)}{\partial z} F_{\beta_{u_{3}}}\left(z_{k}\right) \mathrm{d} z_{k} ; \\
& \left\{Z_{u_{2} u_{2} 22}^{p \alpha_{u_{2}} \beta_{u_{2}}}, Z_{u_{2} u_{2} 66}^{p \alpha_{u_{2}} \beta_{u_{2}}}\right\}=\left\{\widetilde{C}_{22}^{(p)}, \widetilde{C}_{66}^{(p)}\right\} \int_{z_{p}^{b}}^{z_{p}^{t}} F_{\alpha_{u_{2}}}\left(z_{k}\right) F_{\beta_{u_{2}}}\left(z_{k}\right) \mathrm{d} z_{k} ; \\
& Z_{u_{2} u_{2} 44}^{p \alpha_{u_{2} \beta_{u_{2}}}}=\widetilde{C}_{44}^{(p)} \int_{z_{p}^{b}}^{z_{p}^{t}} \frac{\partial F_{\alpha_{u_{2}}}\left(z_{k}\right)}{\partial z} \frac{\partial F_{\beta_{u_{2}}}\left(z_{k}\right)}{\partial z} \mathrm{~d} z_{k} ; \\
& Z_{u_{2} u_{3} 23}^{p \alpha_{u_{2} \beta_{u_{3}}}}=\widetilde{C}_{23}^{(p)} \int_{z_{p}^{b}}^{z_{p}^{t}} F_{\alpha_{u_{2}}}\left(z_{k}\right) \frac{\partial F_{\beta_{u_{3}}}\left(z_{k}\right)}{\partial z} \mathrm{~d} z_{k} ; \\
& Z_{u_{2} u_{3} 44}^{p \alpha_{u_{2} \beta_{u_{3}}}}=\widetilde{C}_{44}^{(p)} \int_{z_{p}^{b}}^{z_{p}^{t}} \frac{\partial F_{\alpha_{u_{2}}}\left(z_{k}\right)}{\partial z} F_{\beta_{u_{3}}}\left(z_{k}\right) \mathrm{d} z_{k} ;
\end{aligned}
$$

$$
\begin{aligned}
& \left\{Z_{u_{3} u_{3} 44}^{p \alpha_{u_{3}} \beta_{u_{3}}}, Z_{u_{3} u_{3} 55}^{p \alpha_{u_{3}} \beta_{u_{3}}}\right\}=\left\{\widetilde{C}_{44}^{(p)}, \widetilde{C}_{55}^{(p)}\right\} \int_{z_{p}^{b}}^{z_{p}^{t}} F_{\alpha_{u_{3}}}\left(z_{k}\right) F_{\beta_{u_{3}}}\left(z_{k}\right) \mathrm{d} z_{k} ; \\
& Z_{u_{3} u_{3} 33}^{p \alpha_{u_{3} \beta_{u_{3}}}}=\widetilde{C}_{33}^{(p)} \int_{z_{p}^{b}}^{z_{p}^{t}} \frac{\partial F_{\alpha_{u_{3}}}\left(z_{k}\right)}{\partial z} \frac{\partial F_{\beta_{u_{3}}}\left(z_{k}\right)}{\partial z} \mathrm{~d} z_{k}
\end{aligned}
$$

Kernels $K_{u_{i} u_{j}}^{p \alpha_{u_{i}} \beta_{u_{j}}}$ for the linear stiffness matrix and Navier solution

$K_{u_{1} u_{1}}^{p \alpha_{u_{1}} \beta_{u_{1}}}=\left(\frac{\pi}{L_{x}}\right)^{2} Z_{u_{1} u_{1} 11}^{p \alpha_{u_{1} \beta_{u_{1}}}}+\left(\frac{\pi}{L_{y}}\right)^{2} Z_{u_{1} u_{1} 66}^{p \alpha_{u_{1}} \beta_{u_{1}}}+Z_{u_{1} u_{1} 55}^{p \alpha_{u_{1}} \beta_{u_{1}}}$

$K_{u_{1} u_{2}}^{p \alpha_{u_{1}} \beta_{u_{2}}}=\left(\frac{\pi^{2}}{L_{x} L_{y}}\right) Z_{u_{1} u_{2} 12}^{p \alpha_{u_{1}} \beta_{u_{2}}}+\left(\frac{\pi^{2}}{L_{x} L_{y}}\right) Z_{u_{1} u_{2} 66}^{p \alpha_{u_{1}} \beta_{u_{2}}}$

$K_{u_{1} u_{3}}^{p \alpha_{u_{1}} \beta_{u_{3}}}=-\left(\frac{\pi}{L_{x}}\right) Z_{u_{1} u_{3} 13}^{p \alpha_{u_{1}} \beta_{u_{3}}}+\left(\frac{\pi}{L_{x}}\right) Z_{u_{1} u_{3} 55}^{p \alpha_{u_{1}} \beta_{u_{3}}}$

$K_{u_{2} u_{2}}^{p \alpha_{u_{2}} \beta_{u_{2}}}=\left(\frac{\pi}{L_{y}}\right)^{2} Z_{u_{2} u_{2} 22}^{p \alpha_{u_{2}} \beta_{u_{2}}}+\left(\frac{\pi}{L_{x}}\right)^{2} Z_{u_{2} u_{2} 66}^{p \alpha_{u_{2}} \beta_{u_{2}}}+Z_{u_{2} u_{2} 44}^{p \alpha_{u_{2}} \beta_{u_{2}}}$

$K_{u_{2} u_{3}}^{p \alpha_{u_{2}} \beta_{u_{3}}}=-\left(\frac{\pi}{L_{y}}\right) Z_{u_{2} u_{3} 23}^{p \alpha_{u_{2}} \beta_{u_{3}}}+\left(\frac{\pi}{L_{y}}\right) Z_{u_{2} u_{3} 44}^{p \alpha_{u_{2}} \beta_{u_{3}}}$

$K_{u_{3} u_{3}}^{p \alpha_{u_{3} \beta_{u_{3}}}}=\left(\frac{\pi}{L_{x}}\right)^{2} Z_{u_{3} u_{3} 55}^{p \alpha_{u_{3}} \beta_{u_{3}}}+\left(\frac{\pi}{L_{y}}\right)^{2} Z_{u_{3} u_{3} 44}^{p \alpha_{u_{3}} \beta_{u_{3}}}+Z_{u_{3} u_{3} 33}^{p \alpha_{u_{3}} \beta_{u_{3}}} ;$

Kernels $K_{G u_{i} u_{i}}^{p \alpha_{u_{i}} \beta_{u_{i}}}$ for the geometric stiffness matrix and Navier solution

$K_{G u_{1} u_{1}}^{p \alpha_{u_{1}} \beta_{u_{1}}}=\left(\frac{\pi}{L_{x}}\right)^{2} Z_{E u_{1} u_{1} 11}^{p \alpha_{u_{1}} \beta_{u_{1}}} ; \quad K_{G u_{2} u_{2}}^{p \alpha_{u_{2} \beta_{u_{2}}}}=\left(\frac{\pi}{L_{x}}\right)^{2} Z_{E u_{2} u_{2} 11}^{p \alpha_{u_{2}} \beta_{u_{2}}}$

$K_{G u_{2} u_{2}}^{p \alpha_{u_{2} \beta_{u_{2}}}}=\left(\frac{\pi}{L_{x}}\right)^{2} Z_{E u_{3} u_{3} 11}^{p \alpha_{u_{3}} \beta_{u_{3}}}$

\section{References}

[1] Carlsson LA, Kardomateas GA. Structural and failure mechanics of sandwich composites. Springer; 2011.

[2] Daniel IM, Gdoutos EE, Wang K-A, Abot JL. Failure modes of composite sandwich beams. Int J Damage Mech 2002;11:309-34.

[3] Vadakke V, Carlsson LA. Experimental investigation of compression failure mechanisms of composite faced foam core sandwich specimens. J Sandwich Struct Mater 2004;6:327-42.

[4] Fleck NA, Sridhar I. End compression of sandwich columns. Composites Part A 2002:353-9.

[5] Fagerberg L. Wrinkling and compression failure transition in sandwich panels. J Sandwich Struct Mater 2004:6:129-44.

[6] Davidson P, Waas AM, Yerramalli CS. Experimental determination of validated, critical interfacial modes I and II energy release rates in a composite sandwich panel. Compos Struct 2012:94:477-83.

[7] Hoff NJ, Mautner SE. Buckling of sandwich type panels. J Aeronaut Sci $1945 ; 12: 285-97$.

[8] Allen HG. Analysis and design of structural sandwich panels. Pergamon Press; 1969.

[9] Frostig Y, Baruch M. High-order buckling analysis of sandwich beams with transversely flexible core. J Eng Mech 1993;119:476-95.

[10] Niu K, Talreja R. Modeling of wrinkling in sandwich panels under compression. J Eng Mech 1999;125:875-83.

[11] Léotoing L, Drapier S, Vautrin A. First applications of a novel unified model for global and local buckling of sandwich columns. Eur J Mech Solids 2002;21:683-701.

[12] Bažant ZP, Beghini A. Sandwich buckling formulas and applicability of standard computational algorithm for finite strain. Composites Part B 2004:35:573-81.

[13] Ji W, Waas AM. Global and local buckling of a sandwich beam. J Eng Mech 2007;133:230-7. http://dx.doi.org/10.1061/(ASCE)0733-9399(2007) 133:2 (230).

[14] Roque CMC, Ferreira AJM, Neves AMA, Mota Soares CM, Reddy JN, Jorge RMN. Transient analysis of composite and sandwich plates by radial basis functions. J Sandwich Struct Mater 2011;13:681-704.

[15] D'Ottavio M, Polit O. Linearized global and local buckling analysis of sandwich struts with a refined quasi-3D model. Acta Mech 2015;226:81-101.

[16] Ley RP, Lin W, Mbanefo U. Facesheet wrinkling in sandwich structures. Technical Report NASA/CR-1999-208994, NASA; 1999.

[17] Hunt GW, Wadee MA. Localization and mode interaction in sandwich structures. Proc R Soc London A 1998;454:1197-216. 
[18] Léotoing L, Drapier S, Vautrin A. Nonlinear interaction of geometrical and material properties in sandwich beam instabilities. Int J Solids Struct 2002;39:3717-39.

[19] Coman CD. On interactive buckling in a sandwich structure. ZAMP 2010;61:565-77.

[20] Hu H, Belouettar S, Potier-Ferry M, Makradi A, Koutsawa Y. Assessment of various kinematic models for instability analysis of sandwich beams. Eng Struct 2011;572-579:2011.

[21] Rammerstorfer FG, Pahr DH, Daxner T, Vonach WK. Buckling in thin walled micro and meso structures of lightweight materials and material compounds. Comput Mech 2006;37:470-8.

[22] Yu K, Hu H, Chen S, Belouettar S, Potier-Ferry M. Multi-scale techniques to analyze instabilities in sandwich structures. Compos Struct 2013;96:751-62.

[23] Arbocz J, Hilburger MW. Toward a probabilistic preliminary design criterion for buckling critical composite shells. AIAA J 2005;43:1823-7.

[24] Fagerberg L, Zenkert D. Imperfection-induced wrinkling material failure in sandwich panels. J Sandwich Struct Mater 2005;7:195-219.

[25] Beghini A, Bažant ZP, Waas AM, Basu S. Postcritical imperfection sensitivity of sandwich or homogenized orthotropic columns soft in shear and in transverse deformation. Int J Solids Struct 2006;43:5501-24.

[26] Bažant ZP. A correlation study of incremental deformations and stability of continuous bodies. J Appl Mech 1971;38:919-28.

[27] Bažant ZP, Cedolin L. Stability of structures. Dover Publications; 2003.

[28] Bažant ZP, Beghini A. Which formulation allows using a constant shear modulus for small-strain buckling of soft-core sandwich structures. J Appl Mech 2005;72:785-7.

[29] Ji W, Waas AM, Bažant ZP. Errors caused by non-work-conjugate stress and strain measures and necessary corrections in finite element programs. J Appl Mech 2010;77. 044504-044504-5.

[30] Ji W, Waas AM. 2D elastic analysis of the sandwich panel buckling problem: benchmark solutions and accurate finite element formulations. ZAMP 2009;61:897-917.

[31] Ji W, Waas AM. Accurate buckling load calculations of a thick orthotropic sandwich panel. Compos Sci Tech 2012;72:1134-9.

[32] Noor AK, Peters JM, Burton WS. Three-dimensional solutions for initially stressed structural sandwiches. J Eng Mech 1994;120:284-303.

[33] Ji W, Waas AM. Wrinkling and edge buckling in orthotropic sandwich beams. J Eng Mech 2008;134:455-61. http://dx.doi.org/10.1061/(ASCE)0733-9399 (2008) 134:6(455).

[34] Kardomateas GA. An elasticity solution for the global buckling of sandwich beams/wide panels with orthotropic phases. J Appl Mech 2010;77 (021015):1-7. http://dx.doi.org/10.1115/1.3173758.

[35] Reddy JN. Mechanics of laminated composite plates and shells: theory and analysis. 2nd ed. Boca Raton, FL: CRC Press; 2004.

[36] Noor AK, Burton WS. Assessment of computational models for sandwich panels and shells. Comput Methods Appl Mech Eng 1995;124:125-51.

[37] Noor AK, Burton WS. Computational models for sandwich panels and shells. Appl Mech Rev 1996;49:155-99.
[38] Carrera E. An assessment of mixed and classical theories on global and loca response of multilayered orthotropic plates. Compos Struct 2000;50:183-98.

[39] Carrera E. Theories and finite elements for multilayered plates and shells: a unified compact formulation with numerical assessment and benchmarking. Arch Comput Methods Eng 2003;10:215-96.

[40] Reddy JN. An evaluation of equivalent-single-layer and layer-wise theories of composite laminates. Compos Struct 1993;25:21-35.

[41] Murakami H. Laminated composite plate theory with improved in-plane response. J Appl Mech 1986;53:661-6.

[42] Carrera E. On the use of Murakami's zig-zag function in the modeling of layered plates and shells. Comput Struct 2004;82:541-54.

[43] Demasi L. $\infty^{3}$ hierarchy plate theories for thick and thin composite plates: the generalized unified formulation. Compos Struct 2008;84:256-70.

[44] D'Ottavio M. A sublaminate generalized unified formulation for the analysis of composite plates. Compos Struct 2016;142:187-99.

[45] Reddy JN. A generalization of two-dimensional theories of laminated composite laminates. Commun Appl Numer Methods 1987;3:173-80.

[46] Washizu K. Variational methods in elasticity and plasticity. 2nd ed. Pergamon Press; 1975.

[47] Carrera E. Theories and finite elements for multilayered plates and shells: a unified compact formulation with numerical assessment and benchmarking. Arch Comput Methods Eng 2003;10:215-96.

[48] Demasi L. $\infty^{6}$ mixed plate theories based on the generalized unified formulation. Part I: governing equations. Compos Struct 2009;87:1-11.

[49] Demasi L. $\infty^{6}$ mixed plate theories based on the generalized unified formulation. Part II: layerwise theories. Compos Struct 2009;87:12-22.

[50] Vescovini R, Dozio L. Exact refined buckling solutions for laminated plates under uniaxial and biaxial loads. Compos Struct 2015;127:356-68.

[51] Hadi BK, Matthews FL. Development of Benson-Mayers theory on the wrinkling of anisotropic sandwich panels. Compos Struct 2000;49:425-34.

[52] Phan CN, Kardomateas GA, Frostig Y. Global buckling of sandwich beams based on the extended high-order theory. AIAA J 2012;50:1707-16.

[53] Phan CN, Bailey N, Kardomateas GA, Battley MA. Wrinkling of sandwich wide panels/beams based on the extended high-order sandwich panel theory: formulation, comparison with elasticity and experiments. Arch Appl Mech 2012;82:1585-99.

[54] Hohe J, Librescu L. Advances in the structural modeling of elastic sandwich panels. Mech Adv Mater Struct 2004;11:395-424.

[55] Dawe DJ, Yuan WX. Overall and local buckling of sandwich plates with laminated faceplates, part I: analysis. Comput Methods Appl Mech Eng 2001;190:5197-213.

[56] Huang H, Kardomateas GA. Buckling and initial postbuckling behavior of sandwich beams including transverse shear. AIAA J 2002;40:2331-5.

[57] Beghini A, Bažant ZP. Stability and finite strain of homogenized structures soft in shear: Sandwich or fiber composites, and layered bodies. Int J Solids Struct 2006;43:1571-93. 